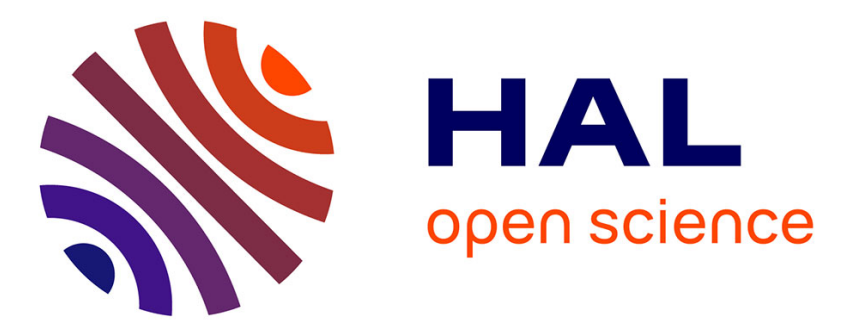

\title{
De l'adversaire religieux à celui du contraire parti. Désigner son adversaire durant les guerres de religion \\ Pierre-Jean Souriac
}

\section{To cite this version:}

Pierre-Jean Souriac. De l'adversaire religieux à celui du contraire parti. Désigner son adversaire durant les guerres de religion. Énoncer / dénoncer l'autre. Discours et représentations du différend confessionnel à l'époque moderne, Brepols, pp.223-242, 2012, 10.1484/M.BEHE-EB.4.00346 . halshs00828410

\section{HAL Id: halshs-00828410 \\ https://shs.hal.science/halshs-00828410}

Submitted on 31 May 2013

HAL is a multi-disciplinary open access archive for the deposit and dissemination of scientific research documents, whether they are published or not. The documents may come from teaching and research institutions in France or abroad, or from public or private research centers.
L'archive ouverte pluridisciplinaire HAL, est destinée au dépôt et à la diffusion de documents scientifiques de niveau recherche, publiés ou non, émanant des établissements d'enseignement et de recherche français ou étrangers, des laboratoires publics ou privés. 


\title{
DE L'ADVERSAIRE RELIGIEUX A CELUI DU CONTRAIRE PARTI. DÉSIGNER SON ADVERSAIRE DURANT LES GUERRES DE RELIGION
}

\author{
Pierre-Jean SOURIAC \\ Université Jean Moulin Lyon 3 - LARHRA (UMR 5190)
}

Chrystel BeRnAT, Hubert Bost (dir.), Enoncer/dénoncer l'autre. Discours et représentations du différend confessionnel à l'époque moderne, Brepols, 2012, p. 223-242.

Pour introduire cette réflexion sur la perception de l'adversaire durant les guerres de Religion, voici une remarque que les magistrats municipaux toulousains adressent à leur souverain en août 1568 , alors que les tensions entre catholiques et protestants renaissent dans le royaume. Dans une lettre adressée au roi pour justifier leur refus d'accepter la paix conclue entre Charles IX et les chefs protestants, la paix de Longjumeau, ils posent la question suivante :

\begin{abstract}
Comment pourroyent lesdits habitans vivre en paix avec ceulx qui de long temps et par tous moyens possibles se sont declairees ennemys de votre couronne et de votredite ville et qui ont juré la ruyne d'icelle l'ayant en l'annee mil cinq cens soixante deux prodictoirement [pour prodicieusement] invadée et en icelle faict plusieurs pilleries, sacrilieges, sacagemens, bruslemens, massacres et aultres actes indignes avec si grande rage et furie que sans la resistence de voz fidelles subjectz ils eussent surprinse toute ladite ville et une partye de votre royaulme et despuis s'estans par le moyen de l'edit de paciffication remis dans icelle auroyent par plusieurs foys essayé tous les moyens possibles pour icelle surprendre et ruyner et a ces fins acisté aux assemblees et concistoires tenuz ez villes de Montauban et de Castres, introduict plusieurs personnes incogneues, enfin, voyant tous leurs desseings rompuz et la derniere conjuration descouverte se seroyent absantés et randuz ez villes rebelles ou ilz auroyent deumoure et porté les armes durant les derniers troubles ${ }^{1}$.
\end{abstract}

Les capitouls de Toulouse posent ainsi explicitement la question problématique de I'altérité religieuse en termes politiques et militaires. Ici, pas de considérations sur l'hérésie ou la nature des oppositions confessionnelles, mais le constat d'un comportement séditieux qui justifie la méfiance des Toulousains, qui justifie de fait le climat de guerre civile. Leurs arguments ne sont pas dénués de fondement, car ils se font l'écho de l'expérience de cette guerre civile. Celle-ci repose sur la mémoire de l'année 1562, mémoire d'un coup de main contre l'hôtel de ville mené par les protestants et de la semaine de combat de rue qui a suivi $^{2}$. Le tout s'est soldé par la victoire catholique, mais dans un sentiment de péril sans précédent pour la ville. Dès lors, l'imaginaire catholique s'est développé selon sa rhétorique habituelle: les excès du réformé, auteur de pillerie, sacrilèges, sacagemens..., le

\section{AM Toulouse, AA 16/125.}

2 M. GREENGRASS, «The anatomy of religious riot in Toulouse in may 1562 », Journal d'histoire ecclésiastique, 1983, p. 367-391 ; J. DAVIES, «Persecution and protestantism : Toulouse, 1562-1575 », The Historical Journal, XXII (1979), p. 31-51 ; P.-J. SouRIAC, Une guerre civile, Seyssel, 2008 ; ID., «Les "urgeans" affaires de la ville. Défendre Toulouse durant la première guerre de religion, 1562-1563 », Revue d'histoire urbaine 3 (juin 2001), p. 39-65. 
comportement héroïque du catholique toulousain défenseur de sa ville, du roi et du royaume. Le problème majeur pour les très catholiques Toulousains est le retour en ville du séditieux au bénéfice de l'édit de pacification d'Amboise, en mars 1563 et l'obligation de coexister entre ennemis. La trahison du protestant, en relation avec les foyers ennemis de l'extérieur que sont Montauban et Castres, est une menace permanente.

La dénonciation de l'adversaire porte sur les actes, sans taxinomie particulière pour désigner l'ennemi rangé sous le vocable de " ceux " qui ont fait ceci ou cela. La désignation des catholiques toulousains est ici très prudente, probablement parce qu'ils s'adressent au roi dans un contexte de pacification, certes précaire, mais voulu par le souverain. Cependant, le sentiment qu'inspirent les actions de leurs adversaires, de leurs " ennemis " est sans doute possible radicalement hostile. II convient donc de revenir sur cette désignation de l'ennemi par le prisme des catholiques toulousains aux prises avec l'apparition du protestantisme et l'intrusion de la guerre civile sur leur lieu de vie. Toulouse a vécu quarante ans dans cette menace et ses habitants, ses magistrats surtout, ont déployé toute une histoire de la désignation de l'adversaire mêlant discours officiel et perception de la menace. Or cette désignation a évolué au fil des événements, au fil des générations et en fonction des interlocuteurs. Cette évolution souligne combien la justification de la guerre par la dénonciation de l'autre pouvait être plastique.

\section{La découverte et le rejet de l'autre}

Les débuts de la Réforme à Toulouse ne sont pas très bien connus, mais l'introduction des idées nouvelles est toujours associée aux corps enseignant de l'université toulousaine perçue comme foyer d'humanisme, notamment pour tout ce qui touche au domaine du droit, et par corrélation, foyer d'évangélisme et de pensées nouvelles en matière religieuse ${ }^{3}$. Rabelais, Étienne Dolet et quelques autres sont passés par Toulouse et ont dénoncé l'intransigeance des catholiques les plus radicaux face à tout foyer intellectuel le rapprochant des nouveautés religieuses. L'Église réformée, pour sa part, semble avoir été fondée à Toulouse vers 1558, et véritablement dressée entre 1560 et 1561 . Sa première manifestation publique a lieu au cours de l'hiver 1561, lorsque l'on commence à entendre le chant des Psaumes venant de maisons particulières, et surtout lors des Rameaux de 1561, lorsque les réformés de Toulouse quittent le collège de l'Esquille, le principal collège toulousain, et se mettent à chanter des Psaumes en procession dans les rues de la ville. Peu après, un des prédicateurs catholiques des plus virulents, le cordelier Melchior Flavin, est apostrophé lors d'un prêche dans une église de la ville, traité de menteur par un auditeur qui est immédiatement saisi et tué par la foule assemblée ${ }^{4}$.

L'Église réformée toulousaine compte environ 4000 personnes sur une ville de 50000 âmes, soit une petite minorité. Elle dispose d'un groupe de pasteurs particulièrement dynamiques, puisque c'est de Toulouse que sont partis ceux qui ont fondé ou organisé les Églises de Castres, de Montauban et des principaux foyers régionaux de la Réforme. Elle a assuré une existence politique à cette Église naissante en cherchant à négocier avec Monluc un soutien par le biais d'un pasteur toulousain et en tenant un colloque des Églises méridionales pour délibérer sur le soutien à apporter à Louis de Condé après la prise

3 H. GILLES, Université de Toulouse et enseignement du droit, XIII -XVI $I^{e}$ siècles, Toulouse, 1992 ; A. DELOUME, Histoire sommaire de la Faculté, Toulouse, 1905.

4 Histoire ecclésiastique des Églises Réformées au royaume de France, éd. par J. W. Baum, E. Cunitz et R. Reuss Paris, 1885, t. I, p. 182 et 373. 
d'Orléans au nom des Églises ${ }^{5}$. Suite à la promulgation de l'Édit de Janvier 1562 qui autorise le culte protestant en France, les réformés toulousains construisent un temple dans un faubourg de la cité et montrent la vitalité de leur communauté. L'Église réformée toulousaine est ainsi une réalité institutionnelle, en semi-clandestinité jusqu'à l'Édit de janvier, mais déterminante à l'échelle régionale et susceptible de drainer des fidèles vers son culte. Son installation s'accompagne de prémices de violence sur lesquelles cette étude ne s'arrêtera pas: prêches publics appelant à la haine, émeutes de rue lors de processions, cette gradation vers la violence est récurrente dans les villes françaises, et Toulouse n'a pas échappé à la règle.

C'est alors que les acteurs en présence théorisent une lecture de la situation urbaine de plus en plus dégradée en raison de cet affrontement latent, lui-même imposé par la coexistence de deux communautés rivales. Examinons les propos tenus dans Toulouse au sujet de cette situation pré-insurrectionnelle, en commençant par ceux des magistrats municipaux. Au cours de l'été 1561, les capitouls ont eu écho de dénonciations à la cour venant de milieux toulousains hostiles à leur politique religieuse jugée trop favorable aux protestants, et ils écrivent le 30 juillet à la reine mère pour se justifier en présentant les lignes d'affrontement au sein de la ville :

\section{Madame,}

Depuis que Dieu par sa grace nous mist a la charge et administration de ceste ville de Tholouze, aurions le plus soigneusement et diligement que nous auroit esté possible, travaillé a entretenir les habitans d'icelle en l'obeissance du roy, paix et tranquillité, laquelle grace a Dieu y a esté, et esperons continuer moyennant l'ayde du seigneur Dieu et du roy notre sire et la votre, laquelle recognoissons estre faible et subject a mille calompnies de plusieurs scismatiques et jaloux de l'unyté et bien de ladicte ville, lesquelz soubz couleur de quelque zele qu'ilz pensent avoir, tachent journellement nous calompnier et que pis est, fere division du peuple tholozain, uny, obeyssant et subject a la majesté du roy s'il en y a autre en tout le royaume, mais certains voulant mectre division en la ville sans auctorité publicque, usurpant les parties du magistrat mis par le roy pour le fere obeyr et reverer, auroient en cachette ces jours passez faict enrooller grand nombre de citoyens pour aller remonstrer au roy notre souverain seigneur de leur loy et foy, comme si lesdits habitans n'estoient d'une mesme religion. Nous croyons (Madame) [sic] que tous sont si uniz comme ilz doibvent estre et soubz une foy, loy et d'un roy et qu'il n'estoit besoing ny loisible ausdits particuliers fere telle description qui ne pourroit tendre que a ung grand scisme et division (Madame), nouz vous avons voulu advertir expressement a ce que foy ne soit adjouster a monsieur Lucas Urdes et Jacques Dessus, advocat et bourgeois, par la ville desadvouez ou meilleur partie d'icelle, comme estans partis sans ses permission, memoires, instructions ny ne luy fere recevoir avec les pretendus roolles forgés a leur poste par personnes privees, affin qu'il plaise a votre majesté (Madame), leur remonstrer qu'ilz se doibvent contenir soubz l'obeyssance du roy et du magistrat et non tacher de calompnier icelluy qui par force et incessemment travailhe pour obeyr au roy et entretenir le peuple en tranquillité et a ce aussi qu'il vous plaise tenir nousdits capitoulz et habitans en votre garde comme bons, tres affectionnez serviteurs, faisant fin a ceste presente par tres humble suplication a notre seigneur de vouloir longuement conserver notre roy en son royaume, sancté grace et protection, donner a sa majesté et vous, Madame, tres longue et heureuse vye. De Tholouze le prenultiesme jour du moys de juillet l'an mil $v^{c} \mid x i^{6}$

5 Blaise DE MONLuC, Commentaires, 1521-1576, «La Pléiade », Paris, 1964, p. 476.

$6 \mathrm{BnF}$, Fr. 15 875, fo $101 \mathrm{r}-\mathrm{v}$. 
Les capitouls déplacent le problème de la sphère religieuse à la sphère civique. Ce qui est alors en jeu, ce n'est pas tant la question du choix confessionnel, mais la conséquence d'un affrontement religieux menaçant de scinder la ville en deux parties distinctes. L'identité politique de la ville, le fondement même de l'idéologie de la bonne ville, repose sur l'illusion d'une communauté citadine unifiée autour de privilèges, de droits et de devoirs admis et reconnus par tous. L'ennemi désigné par les capitouls n'est pas l'autre en religion, mais celui qui contourne le pouvoir municipal pour atteindre le roi sans respecter l'émanation de la communauté civique qu'est le consulat. Sous la plume des capitouls toulousains, la question n'est pas de savoir s'il y a des hérétiques en ville, mais plutôt de souligner combien il y a de mauvais citoyens qui font croire au roi que la ville est en état d'insurrection, que les magistrats ne remplissent pas leur mission. Ce sont bien des "schismatiques " qui sont dénoncés ici, mais en terme de communauté civique et non en termes confessionnels. Les capitouls désignent ceux qui plongent Toulouse dans le chaos, contre l'ordre et l'harmonie municipale.

De fait, les magistrats municipaux ne sont pas totalement honnêtes, notamment sur la question du respect de la foi, dans la mesure où, à cette date, l'Église réformée est bien implantée en ville. Ils représentent l'aile modérée des élites urbaines, certains étant même membres de l'Église réformée clandestine. Demeurés catholiques ou séduits par le calvinisme, les membres du collège municipal portent l'opinion du milieu citadin prêt à admettre une coexistence confessionnelle au nom de la tranquillité publique. En accord avec le virage pris par Catherine de Médicis et Michel de L'Hospital durant l'été 1561, ils dénoncent une intransigeance non explicite, mais ouvrant la voie à la sédition. Ils s'opposent à la faction catholique la plus intransigeante qui milite pour une lutte sans merci contre ceux qu'ils considèrent comme des hérétiques.

Or ces catholiques radicaux, bien représentés au sein du Parlement de la ville et au sein du milieu ecclésiastique, répondent à cette lettre par l'intermédiaire du clergé en s'alignant sur les arguments des capitouls toulousains :

\begin{abstract}
Madame,
Pour l'acquict de la volontaire subjection et tres humble fidelité et obeyssance que nous rendrons tousjours (quoy qu'il advienne) [sic] au roy et a votre magesté, nous vous avons escript ces moys de juing et juilhet derniers les scismes, discordz et divisions qui estoient entre les habitans de ceste votre ville de Tholouze, a raison de la diversité et partialité qu'aulcuns des capitoulz et eschevins d'icelle pour la presente année se efforcoient de y semer. Lesquelz long temps eussent mis ceste ville en semblable subjection que sont les villes de Castres, Montauban, Pamiers, Lavaur, Agen, Lectoure, Villefranche en Rouergue et aultres plusieurs villes de ce pays, si n'eust pleu au roy et a votre magesté de y pourveoir mesmement par l'edict du moys de juilhet dernier et aussi par la commission et mandement qu'il a pleu a sa magesté donner a monsieur de Terride ${ }^{7}$ de y venir, affin de retenir tout le peuple en l'obeyssance de sa magesté avecq une bonne pacification. A quoy il a commencé donner ordre si bon, que nous avons esperance (Dieu aydant) que en peu de temps par sa bonne conduicte, prudence et dexterité, tant ceste ville que tout le pays circonvoisin sera remis en repos et tranquilité, ce qui nous a faict prendre la hardiesse de recourir a vostre magesté pour vous supplier, tres humblement, vouloir tant faire de grace,
\end{abstract}

\footnotetext{
7 Antoine de Lomagne, baron de Terride. Capitaine des ordonnances ayant combattu en Italie, il reçut commission pour aller pacifier Toulouse le 6 août 1561 et eut du mal à se faire recevoir par les capitouls. Par la suite, il participa au siège de Montauban en 1562 qui se solda par un échec. Il fut envoyé en Béarn en 1569 comme lieutenant général des armées du roi, capturé lors du siège de Navarrenx et il mourut peu après en détention.
} 
faveur et humanité a votre pouvre peuple de votre ville et pays criconvoisin que de faire continuer ledit sieur de Terride en la charge jusques a tant que ces troubles soient cessez. Nous avons entendu que lesdits capitoulx sont apres pour le faire revocquer et ont depputé le secretaire La Borniere, pour aller devers sa magesté et la votre pour cest effect, mais nous sommes asseurez, Madame, que sa magesté et la votre aurez plus grande asseurance de la fidelité et saige conduicte dudit sieur de Terride des long temps experimenté que desdits capitoulz qui ont mys tout ce pays en bien fort grand trouble et division, et cause la maladie a laquelle n'est pas en leur puissance de donner remede. Et pour aultant, Madame, que nous sommes asseurez que votre premier desir est de veoir la fin des troubles, divisions et scismes qui sont en ce royaulme tres chrestien, nous vous supplions tres humblement prendre de bonne part et recepvoir l'advertissement et tres humble supplication que nous vous faisons pour la tranquilité, union et pacification de ceste votre ville de Tholouze et tout votre pays adjacent et vouloir de votre benigne grace, humanité et bonté, les exempter de semblables desordres que se commectent journellement esdites villes de Montauban, Lectoure, Lavaur, Castres et aultres et en repoz de noz espritz, nous continuerons prier le createur pour la prosperité et felicité de votre magesté en votre esglise de Tholouze. Le viii jour de septembre $1561^{8}$.

Comme les capitouls, les représentants du clergé arguent du danger de schisme urbain pour fustiger la situation. Seulement sous leur plume, ce danger n'est pas provoqué par les mêmes causes. Au cœur de leur critique se tient le magistrat municipal, garant théorique de l'ordre, mais ne faisant pas son travail en encourageant les dissensions parmi les citoyens. Les capitouls sont accusés de diversité et de partialité. La lettre du clergé catholique n'accuse donc pas ouvertement les capitouls d'être des hérétiques, là aussi probablement parce qu'une telle argumentation n'aurait pas été recevable à la cour à l'automne 1561. La désignation de l'adversaire recourt alors au même vocable que celui qu'on a vu sous la plume du milieu modéré, le schismatique: les magistrats urbains seraient incapables de maintenir l'ordre et l'obéissance au roi en ville en encourageant la division de la communauté civique. Or ici, le motif de division renvoie implicitement à la pluralité religieuse que les capitouls introduiraient ou laisseraient se répandre. Le schisme ne viendrait pas d'une perturbation de l'ordre politique urbain, mais bien d'une altération de l'unanimisme religieux. Avec des mêmes mots, les adversaires toulousains renvoient à des acceptions très différentes du rôle de l'adversaire.

Ainsi, les adversaires toulousains du début de la décennie 1560 se taxent mutuellement de schisme. Dans un contexte politique où se préparent à Paris les premiers textes sur la coexistence, la désignation de l'ennemi religieux ne peut s'appuyer sur des catégories confessionnelles. Du coup, c'est l'ordre public qui est invoqué, et dans ce cas précis, l'unité de la communauté civique, quasiment mythifiée, pour permettre à chaque faction de rejeter sur l'autre la responsabilité de la discorde urbaine. En amont de ces dénonciations demeure la question de la coexistence religieuse dans un même lieu, mais en termes d'expression, seul le vocabulaire de l'ordre public est avancé. Avant même de commencer, les guerres de Religion sont déjà des guerres civiles.

Si cette désignation de l'adversaire, somme toute modérée, correspond au discours municipal adressé à un monarque en quête de conciliation, localement, le positionnement s'avère bien plus dur et le discours anti-hérétique bien plus marqué que dans cette requête. Voici un des multiples arrêts du parlement de Toulouse rendu entre 1560 et 1562 contre les

8 BnF, Fr. 15 875, fo 172 r-v. 
conventicules et hérétiques qui selon lui, pullulent dans la région. Celui-ci est du 10 mai 1560 :

La cour deuement advertie des assemblees, congregations et conventicules cy devant faicts et que se font encore en plusieurs villes et lieux du ressort d'icelle en publicq et en particulier et tant de jour que de nuict contre l'honneur de Dieu et de son Eglise, mespris des edictz du roy, arrestz et deffences cy devans faictes par auctoritée de la court par aucuns desvoyés de notre foy et religion chrestienne par le moyen principallement d'aucuns predicans soy disans ministres, preschans publiquement et en privé, desvoyant et deduysans le peuple par leurs faulces dampnees reprouvees doctrines et changeant l'ordre et fourme des baptesmes, mariages, sepultures autrement qu'il n'est ordonné, institué et receu par notre mere saincte Esglise et aussi a cause du port, vente et distribution de plusieurs livres reprouvés que journellement sont apportez de Genesve et autres lieux suspectz, et voulant pourvoir a telz desordres, inconvenians et scandalles, faire vivre les subjectz du roy en l'amour et crainte de Dieu, ordonnances et constitutions de son Eglise, paix et tranquillité et en l'obeyssance dudit seigneur, ouy sur ce le procureur general du roy et ayant esgard a la requeste par luy sur ce baillee, a ordonné et ordonne que les inquisitions ja commencees quant ausdits assemblees, congregations, conventicules, emotions et port d'armes seront continues par les commissaires ja depputés ou autres que seront cy apres comis et aussi contre ceulx qui font, affichent et placardent libelz fameux et diffamatoires, pour l'inquisition ou inquisitions que sur ce seront faictes par ladite court, veues estre procedé contre les coulpables ainsin qu'il apartient et le cas requerra et cependant exhorte ladite court et par tant que besoing est enjoinct aux archevesques, evesques, premiers curez et autres ayans charge d'ames au ressort d'icelle, prescher et anuncer ou bien faire prescher et anuncer la parolle de Dieu par personnages scavans de bonne vie et sans doctrine, neanlmoings faire fere et continuer le divin service en leurs eglises et parroisses et administrer les sainctz sacremens sans rien innover ains comme il a esté institué et ordonné, gardé et observé par notre mere saincte esglise, enjoingnant a tous subiectz du roy de quelque estat, qualité et condition que soient, d'aller aux esglises, parroisses et autres lieux a ce destinez et ordonnés, ouyr les messes, presches et autres divins services a peine d'estre dictz et declairés suspectz $d$ 'heresie, rebelles et desobeyssans au roy et à justice et a prohibé et deffendu, defend et prohibe ladite court scavoir est ausdits ministres et leurs semblables soys ingerer, prescher en public ou en privé de jour ne de nuict, moings alterer ne abuser desdits sainctz sacremens de baptesme, mariage et autres et a toutes personnes de quelque estat condition et qualité que soient... ${ }^{9}$

La désignation de l'adversaire se fait ici plus précise quant aux rapports de force religieux dans le Midi français, et le Parlement de Toulouse affiche ouvertement le lien qu'il établit entre obéissance au roi et fidélité à l'Église romaine. L'adversaire se voit désigné par divers comportements déviants: participation à des assemblées publiques ou privées totalement illicites, culpabilité en regard de la loi, prédicants et dispensateurs de fausses doctrines, coupables de port d'armes...

Cet arrêt n'est pas le seul en son genre, et le tableau récapitulatif des arrêts toulousains en ce domaine le prouve.

9 AM Toulouse, AA 18/71, p. 105-106. 
Arrêts du Parlement de Toulouse (1560-1587)

\begin{tabular}{|c|c|c|c|}
\hline Date & Motifs de l'arrêt & Requérant & Cote (fonds) \\
\hline 10 mai 1561 & $\begin{array}{l}\text { Contre les assemblées et conventicules } \\
\text { hérétiques; règlement général }\end{array}$ & sprocureur général & $\begin{array}{l}\text { AM Toulouse AA18/71, } \\
\text { 0.105-106 }\end{array}$ \\
\hline 20 août 1562 & $\begin{array}{l}\text { Contre les séditieux et protestants en } \\
\text { armes }\end{array}$ & Procureur général & $\begin{array}{l}\text { bid., AA } 18 / 97, \text { p. } 135 \\
136\end{array}$ \\
\hline 20 sept. 1562 & Contre les séditieux en armes & Procureur général & bid., AA 18/101, p. 139 \\
\hline 27 mai 1565 & $\begin{array}{l}\text { Contre les contrevenants à la paix } \\
\text { Limitation du culte réformé }\end{array}$ & Procureur général & $\begin{array}{l}\text { bid., AA } 18 / 116, \text { p. } 148 \\
150\end{array}$ \\
\hline $\begin{array}{l}1^{\text {er }} \text { octobre } \\
1567\end{array}$ & Contre les protestants en armes* & Procureur général & $\begin{array}{l}\text { AD Haute-Garonne, } \\
\text {-3494/35 (Comminges) }\end{array}$ \\
\hline $\begin{array}{l}11 \text { octobre } \\
1567\end{array}$ & Contre les séditieux en armes & Procureur général & $\begin{array}{l}\text { Ibid., C3494/36 } \\
\text { (Comminges) }\end{array}$ \\
\hline 27 juillet 1568 & Contre les séditieux en armes & $\begin{array}{l}\text { Procureur général } \\
\text { syndic des } \\
\text { capitouls }\end{array}$ & $\begin{array}{l}\text { AM Toulouse, } \\
\text { AA 18/205, p. } 226\end{array}$ \\
\hline 2 août 1568 & Contre les protestants en armes* & $\begin{array}{l}\text { Procureur général } \\
\text { syndic des } \\
\text { capitouls }\end{array}$ & $\begin{array}{l}\text { AM Toulouse, AA } \\
18 / 206, \text { p. } 226-227 \\
\text { AD Haute-Garonne, } \\
\text {-3498/49 (Comminges) }\end{array}$ \\
\hline $\begin{array}{l}10 \text { octobre } \\
1572\end{array}$ & Contre les séditieux en armes & Procureur général & $\begin{array}{l}\text { AM Toulouse, AA } \\
18 / 296, \text { p. } 300-301\end{array}$ \\
\hline 14 août 1573 & Contre les malfaiteurs et pillards & Procureur général & $\begin{array}{l}\text { AD Haute-Garonne, } \\
\text {-3540/29 (Comminges) }\end{array}$ \\
\hline $\begin{array}{ll}19 & \text { janvier } \\
1580 & \end{array}$ & $\begin{array}{l}\text { Contre les contrevenants à l'édit de } \\
\text { paix* }\end{array}$ & Procureur général & AD Tarn, EE 50 (Albi) \\
\hline 14 sept. 1583 & $\begin{array}{l}\text { Contre les perturbateurs du repos } \\
\text { public* }\end{array}$ & Procureur général & $\begin{array}{l}\text { (bid., Edt43, EE } 10 \\
\text { (Cordes) }\end{array}$ \\
\hline 1587 & Contre les courses des voleurs & Procureur général & $\begin{array}{l}\text { AD Haute-Garonne, } \\
\text {-3632/1-8 (Comminges) }\end{array}$ \\
\hline
\end{tabular}

* Documents trouvés sous forme de placard imprimé [tous chez Arnaud ou/et Jacques Colomiez, imprimeurs toulousains.

Les adversaires de la cour sont pour l'essentiel des séditieux, plus que des protestants, même si la désignation religieuse n'est pas absente de ce corpus réglementaire, sous le vocable de "religion prétendue réformée ". Ainsi, le Parlement désigne l'adversaire à combattre et utilise son autorité de cour souveraine pour définir l'ennemi. Son intention est de lancer les catholiques de son ressort contre l'adversaire qu'il désigne ainsi de manière explicite, avec un glissement sémantique sensible entre l'hérétique genevois et le séditieux rebelle à l'autorité royale. Sur ce point, le rôle de la cour souveraine est considérable dans le déclenchement de la guerre civile, dans l'encouragement à la prise d'armes, en rendant des arrêts de règlement qui donnent un vernis légal à la désignation de l'adversaire.

Nous sommes ici dans un débat politico-confessionnel classique, et le Parlement, en utilisant la loi royale, favorise un combat religieux destiné à affaiblir l'adversaire protestant. Comparés aux lettres adressées à Catherine de Médicis, ces textes révèlent les différences dans le vocabulaire utilisé selon les interlocuteurs dans un contexte pourtant identique. La désignation de l'adversaire est donc fluctuante, mêlant altérité religieuse, perturbation de 
l'ordre social et division de la communauté. Selon les interlocuteurs et la finalité du texte, l'un de ces aspects est favorisé.

\section{Désigner l'ennemi militaire}

Le basculement de cette situation vers l'affrontement militaire se fait début mai 1562, suite au coup de force évoqué plus haut. Dès lors, la ville, purgée de ses éléments protestants et modérés, y compris les magistrats municipaux en place au moment de la tentative militaire protestante, devient une citadelle d'un catholicisme partisan engagé dans un conflit totalement assumé. La désignation de l'ennemi change, puisqu'il ne s'agit plus d'un citoyen ayant droit de cité, mais de l'individu contre lequel on a pris et on prendra à l'avenir les armes. Il faut alors nommer le soldat qui pourrait venir assiéger Toulouse ou la surprendre de l'intérieur. S'impose ainsi un vocabulaire militaire qui s'ajoute aux seules désignations religieuses et citadines.

\section{La question des suspects}

Dans le cadre des opérations militaires, la ville engage un processus de purge durant une quinzaine d'années. L'ennemi est alors le suspect, celui qui habite à l'intérieur de la ville et qui menace l'ordre catholique, cinquième colonne prête à agir par traîtrise. Tous ceux que les magistrats au pouvoir jugent de potentiels opposants à l'engagement catholique sont systématiquement désignés, puis pourchassés au nom du devoir civique. Une première entreprise d'épuration est menée au lendemain même des émeutes de mai 1562 : du 25 mai au 17 décembre 1562, le Parlement de Toulouse prononce 1128 condamnations $^{10}$. Le motif officiel de ces arrestations est l'acte séditieux et non la pratique réformée, toujours autorisée selon l'Édit de Janvier. Cependant les magistrats prennent soin de viser ceux qui, de près ou de loin, ont été aperçus aux prêches réformés de la ville. Tous ne sont pas exécutés, loin de là, et si les têtes volent à en croire le très catholique Monluc, la plus grande partie des condamnés trouve refuge à Montauban ou à Castres ${ }^{11}$. C'est là qu'ils attendent l'édit de pacification du printemps 1563 pour tenter de timides retours en ville. Les sept capitouls destitués en mai 1562 et encore survivants mettent plus de trois ans à revenir et plus de dix à récupérer leur dignité et leurs biens; les autres bourgeois aussi bien que les conseillers du Parlement suspects d'hérésie et ayant préféré fuir lors des troubles se trouvent dans une situation tout aussi délicate puisqu'ils subissent des brimades à chaque nouvelle alerte. Ces purges se renouvellent au début de tous les conflits, au moins jusqu'au milieu des années $1570: 1567,1568,1572$ et 1574 sont des années au cours desquelles on dresse des listes de suspects toulousains sont inventoriés. Ceux-ci sont ensuite confinés chez eux pour les femmes, emprisonnés pour les hommes, ou considérés comme fuitifs quand on ne les trouve nulle part ${ }^{12}$.

Ces listes récurrentes créent alors une mémoire partisane fondée à la fois sur une d'anciennes accointances du suspect avec le milieu réformé et le regain systématique d'un

10 J. DAVIES, «Persecution and protestantism », p. 35 ; AM Toulouse, AA 14/1 : liste des personnes dont le Parlement de Toulouse a ordonné l'arrestation et fait saisir les biens du 25 mai au 17 décembre 1562.

11 Blaise DE MonluC, Commentaires, p. 506 : «Et des le lendemain commençarent à faire justice et ne vis jamais tant de testes voler que là. »

12 Joan Davies (voir n. 2) utilise ces listes pour tenter une approche sociologique des milieux réformés toulousains ; voir aussi P. RoMANE-MuSCULUS, «Les protestants de Toulouse en 1568 », Bulletin de la société de l'Histoire du protestantisme français 107 (1961), p. 69-94 ; ID., «Les protestants de Toulouse en 1574 », Bulletin de la société de l'Histoire du protestantisme français 110 (1964), p. 272-283. 
climat de suspicion à chaque prise d'armes. Le poids de cette mémoire ne se lit pas tant dans les listes des suspects que dans les efforts de certains Toulousains pour se faire radier des inventaires des suspects.

Comparution dans le consistoire de la maison de ville, devant les capitouls de Toulouse AM Toulouse, AA15/145, f $^{\circ} 166 \mathrm{v}$

Le 26 mai 1571

François de La Lauze, docteur et avocat de François de Garaut, chevalier, seigneur de Cumières, trésorier de France en la généralité de Toulouse.

Remontre que François de Garaut a obtenu un arrêt du Parlement de Toulouse du 4 mai 1571 aux fins d'estre rayé des deliberations tenues en la maison de la ville des rolles des suspectz, ensemble du livre des mascarades et [demande que soit] pouruivy execution dudit arrest par devant Me. Guillaume Doujac conseiller du roy en la cour...

Examen des précédentes condamnations : procès-verbal des capitouls du 18 nov. 1568 le considérant comme suspect ; inscrit sur les listes de suspects 29 janvier et 18 mars 1569.

Arguments pour sa radiation: éléments de bonne catholicité [baptême des enfants, présence dans son église de paroisse] ; n'a pas pris les armes avec les protestants; a fait fonction de commissaire des vivres dans l'armée de Montmorency-Damville, gouverneur du Languedoc pour le roi, en 1569-1570.

Le suspect est fréquemment associé au "forain ", dont le milieu est soupçonné et systématiquement recherché dans les hôtelleries et refoulé aux portes en cas d'alarme. Les "écoliers", traditionnellement suspectés de désordre et de perméabilité aux idées réformatrices, sont également une cible récurrente. En 1585, " est averti que des écoliers de Toulouse font des assemblées dans les Etudes avec port d'armes et bâtons à feu, dont bon nombre sont suspects; hier [a été vu] sur une heure après midi, plus de 200 écoliers suspects armés de corps de cuirasse, poitrinaux, pistolets et autres, contrevenant ainsi aux édits du roi, arrêts de du Parlement, et interdictions sur ce faites ${ }^{13}$.

Dans ce processus, les habitants sont encouragés à dénoncer leurs voisins, les aubergistes à surveiller leurs hôtes, générant un climat de délation voulu tant par le pouvoir politique que par les autorités religieuses. Il s'agit de définir l'individu à surveiller et exclure, comme le fait en octobre 1567 l'official de la ville qui ordonne d'imprimer et placarder ce texte :

De l'auctorité de monsieur l'official de Tolose, par permission de la Cour souveraine du Parlement de Tolose. A la requeste de monsieur le procureur general du roy, sont admonestés tous coulpables, scavans, consens, donans conseil, faveur, ayde et non revelans du contenu ez articles suyvans

Contre toute personne qui scauroit de certaine science ou autrement que aucuns magistratz et officiers de ce ressort, de quelque qualité ou condition que soient, heussent prinses et acceptés aucunes pensions et gaiges de ceux de la pretendue religion pour tenir la main en la justice, leur servir de defenseurs et protecteurs et qui auroient entendu qui sont lesdictz officiers, et quelles sommes ilz prenoient et la forme du payement d'icelles.

13 AM Toulouse, BB 15, fo 32 v : délibération municipale du 4 mai 1585. 
Qui scauroit aussi de certaine science, ou autrement, aucun conseil estre dressé en ceste ville, pour délibérer des affaires de ladicte pretendue nouvelle religion, et en quel lieu, jour et heure et temps se faisoient lesdictes assemblées et deliberations, entres lesdictz magistratz et officiers, et que aucuns monopolles, conspiration, stipulation de voix et oppinions, aux affaires de justice fussent entre eux dressés et arrestés pour en toutes choses savoir les entreprinses de ceux de ladicte pretendue Religion, contres les edictz et ordonnances du Roy, et que aucuns heussent servy de conseil et juges.

En oultre qui auroit sceu et entendu, par ouy dire ou autrement comme aucuns desdictz seditieux, auroit passé aux maisons particulieres de ceulx de ladicte pretendue Religion, leur donnant advertissement s'en aller hors la ville, et desplacer leurs biens et ce ung peu auparavant ladicte conspiration.

Qui auroit entendu peu de jours auparavant la levée des armes, conspirations, et entreprise contre la majesté du Roy, aucuns propos au tres grand support de ceux de ladicte pretendue Religion avoir esté tenuz de menasses, inthimidations, et jactations d'exterminer les Catholiques, et qu'ilz heussent volonté entreprendre aucune chose contre la majesté $d u$ roy, et estat public de ce Royaume.

Que pour executer ceste tant maligne conspiration, heussent esté dressées aucunes impositions, cotisations, et levées de deniers contre les edictz, et en qu'elle forme, et qui auroient esté les exacteurs d'iceux, et qui sont ceux qui y contribuoient, quel compte en estoit rendu, et quelle forme estoit tenue pour le faire tenir ailheurs, qui estoient ceux qui prenoient la charge de les envoyer et delivrer, et a quelles personnes.

Qui, peu de temps auparavant ladicte conspiration, auroit veu, sceu et entendu, tout en un jour et en diverses maisons avoir esté faicte assemblée, en ceste ville de ceux de ladicte pretendue Religion, principaux, tant Officiers que autres, et le temps qu'ils demeurarent assemblés, et qui estoient assistans ausdictes assemblees.

Tous et chascuns coulpables scavent et consens des choses susdictes ou aulcune d'icelles, l'ayent a reveler devers ledict Seigneur Official, ou maistres Guilleaume de Blancono docteur ez droictz, Simon Pitoys, licentié ez droictz et Jehan Viletart notaire, dans quatre jours apres la publication des presens articles, ou que seront venus à leur notice, sur peine d'excommuniement.

Faict à Tolose le vinghuitiesme iour du moys d'octobre, l'an mil cinq cens soixante sept ${ }^{14}$.

Le contexte est à la reprise des hostilités et les catholiques toulousains ont encore en mémoire le coup de main surprise de mai 1562, où tout avait été préparé en secret par les réformés les plus radicaux sans que les catholiques ne voient venir le coup. L'idée d'une société secrète alliée aux réformés des environs agite alors les autorités catholiques qui encouragent à la délation. Les peurs portent sur les juges traîtres à leur charge, en souvenir de certains conseillers du Parlement rentrés en ville après 1563. Elles portent également sur la tenue en secret d'assemblées clandestines, l'existence d'un réseau préparant un complot et l'organisation d'une " caisse noire " pour financer un éventuel coup de force. II s'agit bel et bien de motifs partisans et militaires.

Il convient de noter enfin qu'à partir de 1574, les ennemis ne sont plus seulement les protestants, mais aussi les catholiques modérés qui, scandalisés par le massacre de la SaintBarthélemy, cherchent à promouvoir une voie médiane, une voie qualifiée de politique. En Languedoc, le gouverneur provincial Henri de Montmorency-Damville se prétendant malcontent de la politique royale, est leur chef de file, et ses proches spnt l'objet de mesures répressives quand ils résident à Toulouse. À partir de 1574, année de l'alliance du gouverneur avec les protestants méridionaux, les notables toulousains proches de

14 AM Toulouse, EE12/129 : ordonnance de l'official du Toulouse, 28 octobre 1567. 
Montmorency-Damville sont inscrits sur la liste des suspects avec le titre de damvilistes ou maréchalistes. Ils ne sont pas véritablement inquiétés pour leur bien et leur vie, mais progressivement écartés de la vie politique de la cité. En 1589 au moment de la Ligue, on retrouve ce même type d'accusation, auxquelles s'ajoutaient celles d'épernonistes et de politiques $^{15}$.

\section{L'ennemi du dehors}

La ville construit alors sa défense face à l'étranger, celui qui vit dans les bastions périphériques tenus par les protestants. On rejoint ici la dénonciation classique de l'assiégé, dans un vocabulaire attesté dans le discours du capitoulat toulousain dès la guerre de Cent $\mathrm{Ans}^{16}$. Ce discours militaire se décompose en trois modalités descriptives :

\section{- L'ennemi :}

Délibération municipale du 16 février $1569^{17}$

S'est presanté noble Francois de Mervielh capitaine de la ville de Villemur qui a dict les ennemys de Dieu et du roy venant de Saint Anthonin auroient faict plusieurs courses de quoy adverty pour le service de sa magesté soict sourty avec plusieurs troppes de souldatz et combatu ou seroient demeures plusieurs et leur auroict gaigné certains balles cuyrs et autres forrages...

- Le pillard :

Délibération municipale du 15 juillet $1585^{18}$

Le Parlement a eu avis qu'aux environs de Toulouse des compagnies de gens de pied et cheval, sorties de Montauban, Castres et Puylaurens et autres villes rebelles et détenues par les hérétiques, suivies d'infinité de volleurs qui sont dessendus des Sevennes et Auvergne qui ce disent estre du roy de Navarre, lesquelz se jactent faire le gast, prendre, ravir et emporter la gerbe et grains qui sont couppés et despicqués et garder que les vraiz catholiques serviteurs de Dieu et du Roy ne fassent la recolte, mesme se ventent mectre le feu aux mesteryes appartenans aux habitans de Tholoze estant resolus de tenir la campagne, vuyder et quitter toutes les villes qui sont a leur devotion non tenables et icelles brusler fors que les villes de Mazeres, Saverdun, Pamyes et Tarrascon.

- L'occupant :

Délibération municipale du 5 mai 1586

harangue de l'évêque de Comminges, Urbain de Saint-Gelais ${ }^{19}$.

Par le moyen de quelques traitres la ville de Sainct Bertrand quy est chef de son evesché a este envahie et prinse par lesdits hereticques et par ce que c'est une ville d'importance quy pourroict pourter grand prejudice a ceste ville [de Toulouse] a cause de la

15 J. DAVIES, «Persecution and protestantism », p. 46 ; A. JouANNA, «Protection des fidèles et fidélité au roi : l'exemple de Henri $\mathrm{I}^{\mathrm{er}}$ de Montmorency-Damville», dans Y. DURAND, Hommages à Roland Mousnier: Clientèles et fidélités en Europe à l'époque moderne, Paris, 1981, p. 279-296 ; M. GREENGRASS, « Noble affinities in Early modern France : the case of Henri $\mathrm{I}^{\mathrm{er}}$ de Montmorency-Damville, constable of France », European History Quarterly (1986), p. 275-311.

16 X. NADRIGNY, «L'information politique à Toulouse dans la première moitié du XIV ${ }^{\mathrm{e}}$ siècle », Thèse de l’École des Chartes, 1999.

17 AM Toulouse, BB 112, feuille volante.

18 AM Toulouse, BB $15, f^{\circ} 82 r$.

19 AM Toulouse, BB 15, f 373 r. 
riviere et autres commodites qu'elle en recoyt et que icelle pour le present n'est munye de provisions ny de forteresse, que si on les y laisse il en pourroict venir mil inconveniens premierement que l'heresie s'y fondera comme es autres provinces, secondement qu'ilz se ranforceront et muniront de munitions tellement que pour y remedier tous les gentilshommes circonvoisins se sont avec le menu peuple leves en armes et sont au devant, mais parce que icelle ville ne peult estre forcee avec la main et sans quelque piece de canon et que ceste ville de Tholouze est la mere nourriciere des autres villes a supplié icelle voulloir assister de quelques pieces de canon.

De tels textes se déclinent à l'infini dans le fonds documentaire toulousain, sous forme de lettres, de comparutions devant les magistrats ou de délibérations entre magistrats euxmêmes. C'est l'ennemi qui est ici désigné, le plus souvent associé à un terme religieux : hérétiques ou ennemis de Dieu. Mais ces désignations religieuses sont automatiques, jamais développées, elles correspondent à une taxinomie de l'adversaire comme on dirait l'Espagnol ou le Tudesque. Si l'adversaire est bien associé à une identité religieuse, cette dernière n'est qu'une désignation générique, qui permet seulement de nommer l'ennemi. En revanche, c'est bien d'un combattant qu'il est question, celui qui tient la campagne et rançonne les paysans, qui occupe une place forte du piémont pyrénéen, avec lequel on s'engage dans des escarmouches.

\section{L'ennemi confessionnel}

À côté de cette désignation militaire, l'identité religieuse de l'adversaire est exprimée à l'occasion de véritables spasmes religieux qui agitent sporadiquement les rues toulousaines. II s'agit d'appels à la croisade, à une ligue, suivie d'une procession, d'un serment, de violences et quelquefois de la levée d'une petite armée. Le temps des guerres de Religion est propice à ces manifestations religieuses dans les rangs catholiques, et le Midi est particulièrement touché ${ }^{20}$. Ces spasmes accompagnent la guerre, mais, s'ils peuvent déboucher sur une intense mobilisation, leur durée est bien plus courte. La foule catholique toulousaine est capable de s'enthousiasmer pour la lutte contre l'hérétique, mais cet élan fait vite place à la routine de la guerre, et l'abominable briseur d'hostie redevient le banal soldat du quotidien. C'est dans ces élans religieux que la guerre se voit justifiée, que les " guerriers de Dieu " puisent la détermination de leurs gestes. Mais leurs élans ne durent guère, justement parce qu'ils sont intenses. La ville de Toulouse connaît de tels événements tout au long des guerres dans le cadre des mouvements associatifs autrement appelés ligueurs.

Les suites des combats de mai 1562 donnent lieu à des manifestations destinées tout autant à expier la faute des citadins passés à l'adversaire qu'à rendre grâce à Dieu pour son aide dans le salut de la cause catholique. À la fin de la première guerre, en mars 1563, une ligue est scellée, initiée par l'archevêque, le cardinal d'Armagnac, le Parlement et les chefs de guerre languedociens Guillaume de Joyeuse et Raymond de Fourquevaux ${ }^{21}$. À la suite des mouvements observés en Bourgogne à la même époque, une croisade est prêchée à

20 K. Gould, Catholic Activism in South-West France, 1540-1570, Ashgate, 2006.

21 AM Toulouse, AA 18, p. 110. Les principaux signataires de cette association étaient le cardinal Georges d'Armagnac, archevêque de Toulouse, Laurent de Strozzi, évêque d'Albi, Blaise de Monluc, lieutenant général du roi en Guyenne, Raymond de Fourquevaux, gouverneur de Narbonne et représentant de Guillaume de Joyeuse, lieutenant général du Languedoc, Antoine de Lomagne, baron de Terride, et Louis de Carmaing, comte de Nègrepelisse capitaines d'une compagnie d'ordonnance, les commissaires du Parlement et les capitouls. 
Toulouse en septembre 1568, suscitant la formation d'une petite armée qui part se battre sur les rives de l'Ariège ${ }^{22}$. Quatre ans plus tard, au début du mois d'octobre 1572, les répercussions provinciales de la Saint-Barthélemy sont l'occasion de perpétrer plus d'une centaine assassinats dans les prisons du Parlement de la ville par un noyau catholique qui compose par la suite le cœur de la faction ligueuse ${ }^{23}$. Toulouse relaie ensuite l'engagement local dans la ligue de Péronne de 1576-1577 et prête le serment demandé par le roi à cette occasion. Elle en fait de même à partir de 1585, avec des serments d'adhésion aux textes ligueurs approuvés par le roi et la structuration d'associations locales visant à combattre les protestants. Enfin, à partir de janvier-février 1589, suite à l'assassinat des Guises à Blois, les Toulousains adhèrent à la Sainte-Union parisienne et permettent le glissement de Toulouse dans la révolte contre Henri III puis Henri IV, jusqu'en janvier $1596^{24}$.

À la différence des précédents discours sur la guerre, les textes de serment développent à l'envi la figure de l'hérétique sanguinaire prêt à massacrer les sujets catholiques du roi vivant à Toulouse. À titre d'exemple, on peut s'arrêter au début de la troisième guerre de Religion, à l'automne 1568, durant lequel Toulouse est agitée par un appel à la croisade. Hector d'Ossun, évêque du Couserans, chassé de son palais par les réformés, vient à Toulouse pour prêcher une guerre sainte contre les hérétiques. Il reçoit un accueil enthousiaste et, le 12 septembre, est scellée dans la cathédrale Saint-Étienne une nouvelle association jurée appelant les fidèles à prendre les armes et à mourir dans le Christ au nom de sa foi et de l'Église.

Les catholiques loyaulx et fidelles subjects du roy, voyant que les heretiques sacramentaires Calvinistes, aprez avoir obtenu grace et paix du roy très-chrétien, deux fois sont recheus en toutz pechez et erreurs qu'ont esté depuis le commencement du monde, jusques à un entier atheisme, vivans sans reconnoistre Dieu, sans foy et sans loy, qui tâchent abolir la connoissance de Dieu et de son fils Jesus-Christ, duquel parlent innominieusement, et de sa très-sacrée mere, et des saints de paradis ; et que pour abolir la memoire de notre Seigneur Dieu eternel avec le Pere, ils abolissent tous les sacremens, les temples, les croix, les crucifix, et toute la memoire de la passion et benefice de notre redemption, bruslent les saints livres, meurtrissent les docteurs et prédicateurs, prestres, chretiens cruellement et inhumainement, et se vantent d'arracher toute la pretrise, et priver le roy de toute sa puissance et autorité ez villes de Montauban et autres villes qui sont sous leur puissance ez pays de Guyenne et de Languedoc ; et les hereticques de la Guyenne, qui ne recognoissent pour roy sinon la royne d'Angleterre, à laquelle appartient le duché de Guyenne, comme ils disent temerairement, et que outre ce dessus, par force violence, ils se sont rendus maîtres de Castres, Puilaurens, Réalmont, Millau, Saint-Antonin, Montauban, Le Carla, Le Masd'Azils, Le Tosat et depuis naguieres ont surpris Gailhac, Mazeres, Carmaing, Auriac, les Catalens, le Bourg-Saint-Bernard, et autres villes mediocres, là où ils ont meurtri tous les chretiens, hommes, femmes, petits enfants ; et non contens de ce, sont si temeraires, qu'ils

22 J. LECLERC, «Aux origines de la Ligue, premiers projets et premiers essais (1561-1570) », Études 227 (1936), p. 203 ; DEVIC - VAISSETTE, Histoire générale du Languedoc, Toulouse, 1889, t. XI, p. 509 ; pour le contenu de l'acte de la croisade, t. XII, col. 888 ; D. CROUZET, Les guerriers de Dieu, Seyssel, 1992, t. I, p. 386389 ; J. DE CARDONNE, Remonstrance aux catholiques de prendre les armes en l'armée de la Croisade instituée en la ville de Tholose contre les calvinistes huguenots, traîtres et rebelles, Toulouse, Colomies, 1568 ; R. SAUZET, Au grand siècle des âmes. Guerre sainte et paix chrétienne en France au XVII siècle, Paris, 2007, p. $81 \mathrm{sqq}$.

23 J. GARRISSON, Tocsin pour un massacre : la saison des Saint-Barthélemy, Paris, 1968, p. 143 ; D. CROUZET, Les guerriers de Dieu, t. II, p. 116.

24 M. GREENGRASS, "The Sainte Union and the provinces: the case of Toulouse », The Sixteenth century journal XIV-4 (1983), p. 469-496. Voir une partie de l'édition des délibérations municipales de 1577 à 1589 dans J. LOUTCHITZKY, Documents inédits pour servir à l'histoire de la Ligue et de la Réforme, Paris, 1873. 
ne font difficulté entreprendre sur la ville de Toulouse, où ils ne veulent laisser personne en vie, jusques que les rues fussent ruisseaux de sang, coulant jusques aux sangles de leurs chevaulx, laquelle perdue le roy perdroit le Languedoc et Provence ; et outre ce, se ventent, que pour ung temps se serviront d'aucuns gentils hommes hereticques et traîtres du roy et république de France; mais aprez veulent ecraser la noblesse et la justice. Nous, voyant cecy depuis ung an, ne cessons prier Dieu, jeuner et recevoir le Saint Sacrement, faire processions avec le corps de Jesus Christ, par quoy notre Seigneur nous inspire nous croiser, et prendre les armes, dresser une guerre comme Mathatias et les autres Macabées, ayant confiance que Dieu sera avec nous, comme il fut à la guerre contre Attila, qui fut deffait pres de Toulouse avec trois cens mille hommes, et comme il fut avec nos bons catholiques à la guerre de Muret, là ou huit cens des notres, deffirent plus de soixante mille hérétiques, au temps de Loys huitieme, père de saint Loys; en sorte que nous ne douptons point que nous n'obtenons la victoire contre ces ennemis de Dieu et de toute nature humaine; et si aulcung de nous y mourrons, notre sang nous sera un second baptême, par quoy sans aucun empeschement, nous irons avec les autres martyrs droit en paradis.

Fait à Thoulouse, ce XII septembre $1568^{25}$.

La désignation de l'adversaire passe ici par une abondance de qualificatifs et d'expressions associant le protestant à l'hérétique: "hérétiques sacramentaires ", " calvinistes ", " atheisme, vivans sans reconnoistre Dieu, sans foy et sans loy, qui tâchent abolir la connoissance de Dieu ", blasphémateurs à l'égard de la vierge Marie, destructeurs des temples et des sacrements. Le texte glisse de l'hérétique à l'athée sans que ces contradictions n'affectent les auteurs. Il en arrive ensuite au révolté contre le roi : "se vantent d'arracher toute la pretrise, et priver le roy de toute sa puissance et autorité», avec une mention de la Guyenne et du passé anglais, allusion anachronique mais toujours utile pour désigner le traître. Les protestants sont ensuite assimilés à des massacreurs : " là [en parlant des villes tenues par les protestants] où ils ont meurtri tous les chretiens, hommes, femmes, petits enfants; et non contens de ce, sont si temeraires, qu'ils ne font difficulté entreprendre sur la ville de Toulouse, où ils ne veulent laisser personne en vie, jusques que les rues fussent ruisseaux de sang, coulant jusques aux sangles de leurs chevaulx ". Dans cette partie du texte, l'allusion est très révélatrice de l'imaginaire catholique. Aucune des villes du Midi toulousain n'ont connu de tels massacres de populations catholiques par les réformés, ce fut plutôt l'inverse, et les villes tenues par les protestants le furent non par des coups de force militaire, mais par un basculement politico-religieux des élites dès les années 1561-1562. Or, dans l'expression de leur angoisse à l'égard des pulsions massacrantes des protestants, les catholiques toulousains retranscrivent non ce que leur communauté a subi, mais ce dont ils ont été témoins du fait de leur propre parti. II s'agit bien plus de leur imaginaire que du comportement potentiel de leurs adversaires. Enfin, pour asseoir leur engagement, plusieurs exemples historiques sont invoqués en vue de comparer la situation présente à des équivalents mémorables : Judas Maccabée (catholiques) contre les séleucides syriens (protestants) ; Attila défait devant Toulouse - ce qui est une falsification historique ; la guerre de Muret, c'est-à-dire la bataille de Muret (12 septembre 1213) durant la croisade des Albigeois. Sur ce dernier point, la comparaison est cocasse, les Toulousains de 1213 étant plutôt du côté des hérétiques que des croisés.

Dans ce registre, l'adversaire est ici bien plus qu'un soldat ; c'est l'ennemi dans la foi contre lequel on se croise, contre lequel on est prêt à mourir au nom de Dieu. Ce discours

25 Devic - VaissetTe, Histoire du Languedoc, t. XII, col. 888-889: première partie du serment prêté à Toulouse le 12 septembre 1568 . 
confessionnel est complémentaire de la description strictement militaire. Tous deux renvoient des formes de mobilisation individuelle différentes, mais liées l'une à l'autre. L'ennemi religieux contre lequel se bat le ligueur ou le croisé est cependant un ennemi exceptionnel, défini par une haine farouche exprimée le temps d'un spasme, d'une émeute, d'une procession, d'un geste de guerre hors norme. II laisse assez rapidement place à une banalisation de la guerre, l'hérétique perdant son caractère d'être exceptionnel condamné à souffrir une vengeance divine immédiate, quand justement cette vengeance divine tarde à venir. II n'est alors pas surprenant de trouver ces spasmes de violence religieuse en début de conflit, alors que par la suite la guerre entraîne une dilution de l'élan initial. L'ennemi reste I’hérétique, mais cette désignation devient générique, perdant de son caractère sacral.

\section{Sortir de la guerre : celui du contraire parti}

Un autre rapport à l'ennemi apparaît au cours de la dernière guerre de Religion, à partir du milieu des années 1580. C'est à cette époque qu'apparaissent des trêves de labourage, mouvement déjà étudié par ailleurs ${ }^{26}$, mais intéressant ici car il traduit un changement d'attitude à l'égard de l'adversaire. Ces accords de trêve n'ont pas l'ambition de sceller un rapprochement entre adversaires, mais de définir une sorte de droit de la guerre. Ils s'inscrivent dans le même esprit que les trêves de Dieu médiévales par lesquelles l'Église circonscrivait l'usage de la violence au sein du milieu nobiliaire en excluant des combats les populations non armées et en interdisant l'usage des armes certains jours de la semaine ou certains mois de l'année ${ }^{27}$. Les trêves de labourage n'ont d'autre ambition que de protéger les civils des combats, mais elles diffèrent des textes médiévaux par les moyens employés et par les autorités contractantes. Ce sont les autorités civiles et militaires, municipales et provinciales, et non les Églises, qui encadrent la mise en place de ces contrats. L'espace politique qui sert de cadre à ces accords était la province : une grande partie des trêves sont négociées directement par les lieutenants généraux et gouverneurs de Languedoc et de Guyenne, ou conduites par les assemblées locales, villes et provinces mais placées sous l'autorité provinciale ${ }^{28}$. En dessous de ces chefs de guerre provinciaux commissionnés par le roi ou les partis, l'application locale des trêves dépend essentiellement des états provinciaux et des villes, donc pour Toulouse, de l'engagement des capitouls.

Ce mouvement apparaît dès 1586, mais les capitouls toulousains le rejoignirent sur le tard, puisque leur première participation directe au nom de la ville remonte à 1590 . On les trouve ensuite de manière récurrente jusqu'à la fin du conflit ${ }^{29}$. Le principe est de limiter la guerre à des domaines très précis et de préserver à cette fin les paysans et le bétail, de permettre la mise en culture des terres en interdisant la destruction des champs et la

26 P.-J. SOURIAC, «Éloigner le soldat du civil en temps de guerre. Les expériences de trêve en Midi toulousain dans les dernières années des guerres de Religion », Revue historique 306-4 (2004), p. 788-818.

27 J. FAVIER, Dictionnaire de la France médiévale, Paris, 1993, p. 715. Dans les dictionnaires de Trévoux ou de Moreri cités précédemment, la notion de trêve est systématiquement associée à celle de trêve de Dieu, signe de la persistance d'une mémoire de ces mesures pacificatrices médiévales.

28 AD Tarn, C 854 : trêve du Languedoc, mars 1588 : comme l'ensemble des trêves, celle-ci est d'abord signée par Guillaume de Joyeuse qui s'engage à l'appliquer et la faire respecter, puis dans les mêmes termes par Henri de Montmorency-Damville, gouverneur du Languedoc sous l'obéissance d'Henri de Navarre ; l'ensemble des pièces était transmis avec ces certificats aux autorités locales, ici albigeoises.

29 Présence de Toulouse aux négociations de trêves : Tarabel, 2 novembre 1590 [AD Hérault, C 8399, et AD Tarn, AA 20, pièce 203, fo530 v] ; Puylaurens, 12 avril 1591 [AD Tarn, C 859] ; Saint-Félix, 24 juin 1592 [AM Toulouse, AA 16, fo275 r-v] ; Grisolles, 7 avril 1593 [AD Hérault, C 8399] ; L’Isle-Jourdain, 25 juin 1593 [AM Toulouse, AA 20, pièce 594, p. 234]. 
capture des paysans. Au début, les textes de trêve ne concernent que le travail de la terre ; progressivement, ils s'étendent à tous les secteurs de la vie économique : marchands, financiers, artisans... La guerre demeure, mais on cherche à la contenir au seul champ de bataille, à en limiter l'incidence civile. Les représentants des deux partis se rencontrent à l'échelle des chefs provinciaux pour les grands principes, à l'échelle des communautés pour l'application locale. Ils se mettent d'accord pour rédiger le contenu de la trêve ou reconduire un texte préexistant. À tous les échelons de la province, des contacts se nouent entre adversaires pour arriver à s'entendre sur un texte que tous souhaitent voir appliquer. On ne raisonne plus alors en termes d'ennemis, d'hérétique ou de papiste, mais en termes de parti :

\section{Assemblée de Puylaurens -12 avril $1591^{30}$}

Les depputes des villes et diocezes de Tholouze, Alby, Lavaur, Sainct Papoul et dioceze Basse de Montauban assembles en la ville de Puylaurens faisant tant pour eux que autres diocezes du hault Languedoc absens d'ung et d'autre party par permission et haultorité tant de monseigneur le duc de Montmorancy, pair et mareschal de France, gouverneur et lieutenant general pour le roy en Languedoc, que de monseigneur de Joyeuse aussy mareschal de France, lieutenant general pour le roy audit pais, pour l'execution, entretenement et observation de la tresve du laboreur et bestail de labouraige et ce quy en deppend, faicte et accordée entre leurs grandeurs le quatriesme de novembre 1590 pour ung an entier, reparation et restitution des contreventions faictes à icelle d'ung et d'autre party à ce qu'a l'advenir elle puisse estre plus exatemant observée et les difficultes et empechemens que interviennent journellement, ouster et pour plus grand esclarcissement et ampliffication de ladite tresve a esté deliberé et arresté et supplier leursdites grandeurs voulloir agreer et authoriser pour le bien et soulaigement du peuple les articles suyvant.

De telles assemblées se retrouvent sur tous les fronts confessionnels du Midi, partout où catholiques et protestants vivent en voisinage immédiat. Ces négociations indiquent une évolution considérable dans la perception de l'altérité religieuse car elles présupposent l'acceptation d'un dialogue avec l'autre. Pendant la durée de la négociation, on cesse de le traiter d'hérétique pour le considérer seulement comme celui du contraire parti. Cette désignation vient se substituer à la taxinomie belliqueuse et fournit à l'adversaire une forme d'identité pacifiée ${ }^{31}$. Ces négociations impliquent un modèle de coexistence imposé par le refus général d'une guerre qui a autant usé les ressources locales qu'émoussé l'ardeur des combattants. Sur le plan religieux, la principale caractéristique des trêves est de ne jamais aborder le problème confessionnel, son ambition n'étant pas d'être une paix de religion, mais un cheminement vers l'éradication de la guerre. On est alors dans un règlement civil du conflit, une limitation de la violence religieuse sur des critères de survie matérielle supérieurs aux arguments théologiques. À cet égard, ces négociations préparent la pacification henricienne à venir et témoignent du refus de plus en plus massif des désordres de la guerre. Pour tenter de garantir l'application de ces trêves, les assemblées nomment

30 AD Tarn, C 859

31 À titre d'exemple : AD Tarn, C 8399 : «Continuation de la procedure du sieur d'Encuns, pour l'observation de la tresve accordée en fevrier 1590, et depuis accordees de nouveau et confirmee entre les villes catholiques limitrophes du comté de Foix, du diocèse de Toulouse et autres avoisinantes et par les villes et lieux du contraire parti du comté de Foix » [livre du commissaire d'application de la trêve pour 1590]. Autre exemple : AD Hérault, C 8389 : négociation de la trêve à Venez, en novembre 1588 : le député du diocèse de Toulouse fait savoir « qu'en son diocèse toutes les villes et lieux fermes se gardent à leurs despens sans impositions generalles que sur eux mesmes et que ceulx du party contraire doibvent faire le semblable » [discussion sur le paiement des garnisons et l'établissement ou non d'une taxe provinciale]. 
deux commissaires, un de chaque bord, qui passent de village en village surveiller les soldats et recueillir les plaintes. Leurs registres éclairent sur la perception de l'adversaire qu'ont alors les Méridionaux : soldats volant du bétail, pillant les greniers ou rançonnant un village. L'ennemi n'est plus le protestant, mais bien la soldatesque, par un retour en force du discours traditionnel des malheurs de la guerre ${ }^{32}$.

Catholiques et protestants se sont affrontés pendant près de quarante ans sans que la perception et la désignation de l'adversaire soit identique selon les acteurs et les temps de cette guerre. L'exemple toulousain souligne la physionomie changeante de l'adversaire, à la fois perturbateur de l'ordre public, royal comme municipal, guerrier redoutable et hérétique blasphémateur. Cette polysémie ne brouille pas le visage de l'adversaire, mais elle permet de s'adapter à la conjoncture et aux attentes des principaux intéressés. Dans un contexte de coexistence imposée par le pouvoir central, l'ennemi est celui qui menace la cité. Dans un contexte de guerre longue et statique, il est ce soldat secondé par des intelligences à l'intérieur de la cité, prêt à frapper à tout moment. Dans la ferveur d'un acte de piété, il est le nouvel Attila qu'il faut terrasser. Il s'agit cependant toujours du même individu, dont la physionomie change selon qu'il faut combattre, prier ou attendre. La véritable rupture n'apparaît qu'en fin de guerre, quand à cette polysémie de l'adversaire se substitue celle de l'alter ego avec lequel il convient de négocier. Ce changement dans le langage et la relation à l'autre indique une maturation de la société toulousaine, son cheminement vers une acceptation de la paix, vers l'établissement d'une coexistence qui ne serait pas source de schisme.

32 À titre d'exemple : AD Tarn, C 855 : Inquisition des commissaires du diocèse d'Albi, 1588. 